\title{
BMJ Open Process mapping evaluation of medication reconciliation in academic teaching hospitals: a critical step in quality improvement
}

\author{
Anne Holbrook, ${ }^{1,2,3}$ James M Bowen, ${ }^{4,5}$ Harsit Patel, ${ }^{6}$ Chris O’Brien, ${ }^{6}$ \\ John J You, ${ }^{2,4,6}$ Roshan Tahavori, ${ }^{7}$ Jeff Doleweerd, ${ }^{8}$ Tim Berezny, ${ }^{8}$ Dan Perri, ${ }^{1,2,5}$ \\ Carmine Nieuwstraten, ${ }^{5}$ Sue Troyan, ${ }^{7}$ Ameen Patel ${ }^{2,6}$
}

To cite: Holbrook A Bowen JM, Patel H, et al. Process mapping evaluation of medication reconciliation in academic teaching hospitals: a critical step in quality improvement. $B M J$ Open 2016;6:e013663. doi:10.1136/bmjopen-2016013663

- Prepublication history for this paper is available online. To view these files please visit the journal online (http://dx.doi.org/10.1136/ bmjopen-2016-013663).

Received 2 August 2016 Revised 23 November 2016 Accepted 30 November 2016

CrossMark

For numbered affiliations see end of article.

Correspondence to Dr Anne Holbrook; holbrook@mcmaster.ca

\section{ABSTRACT}

Background: Medication reconciliation (MedRec) has been a mandated or recommended activity in Canada, the USA and the UK for nearly 10 years. Accreditation bodies in North America will soon require MedRec for every admission, transfer and discharge of every patient. Studies of MedRec have revealed unintentional discrepancies in prescriptions but no clear evidence that clinically important outcomes are improved, leading to widely variable practices. Our objective was to apply process mapping methodology to MedRec to clarify current processes and resource usage, identify potential efficiencies and gaps in care, and make recommendations for improvement in the light of current literature evidence of effectiveness.

Methods: Process engineers observed and recorded all MedRec activities at 3 academic teaching hospitals, from initial emergency department triage to patient discharge, for general internal medicine patients. Process maps were validated with frontline staff, then with the study team, managers and patient safety leads to summarise current problems and discuss solutions.

Results: Across all of the 3 hospitals, 5 general problem themes were identified: lack of use of all available medication sources, duplication of effort creating inefficiency, lack of timeliness of completion of the Best Possible Medication History, lack of standardisation of the MedRec process, and suboptimal communication of MedRec issues between physicians, pharmacists and nurses.

Discussion: MedRec as practised in this environment requires improvements in quality, timeliness, consistency and dissemination. Further research exploring efficient use of resources, in terms of personnel and costs, is required.

\section{BACKGROUND}

Hospital-based medication reconciliation (MedRec) is defined as the process of identifying the most accurate list of a patient's preadmission medications-including the name,

\section{Strengths and limitations of this study}

- The first study to clarify the many steps and roles involved in admission medication reconciliation (MedRec) for complex patients in teaching hospitals.

- Process mapping, an underused methodology in healthcare, can rapidly diagnose inconsistencies, gaps and overlaps in care processes and form the basis of personnel costing studies.

- Our location was limited to one urban area's hospitals ( 1900 beds in total), serving a large region of Ontario, Canada (population 2.3 million; $14000 \mathrm{~km}^{2}$ ).

- The actual cost-effectiveness of MedRec, as currently practised, is a high priority for investigation if it remains a hospital accreditation standard internationally.

- We clarified five general areas where MedRec could be improved for better quality and efficiency.

dosage, frequency route, last dose taken and assessment of adherence, comparing them to the current medication orders, recognising and addressing discrepancies, and documenting any changes, thus resulting in a 'Best Possible Medication History (BPMH)'. MedRec is a component of medication management, which involves the wider and more important evaluation of diagnoses, contraindications, patient values, and evidence for comparative cost-effectiveness of medications to craft and communicate the most appropriate medication regimen for the patient.

In response to a perceived medication safety crisis, MedRec was mandated by national hospital accreditation bodies in Canada and the USA in 2006 as a Required Organizational Practice (ROP), and has been recommended by the National Institute of Health and Care Excellence in the UK. ${ }^{12}$ 
This major change in system responsibility, work flow and healthcare professional priorities, without accompanying personnel or information technology support, was based entirely on literature using avoidance of 'potential medication errors' as a primary outcome. MedRec ROPs in the USA had to be scaled back due to the failure of proponents to recognise the complexity and costs of the process, and hospitals' inability to meet requirements for widespread MedRec across the continuum of care. Similarly, Canadian hospitals are struggling to meet ROPs for admission MedRec with 57.4\% overall compliance, are even further behind on discharge MedRec, and are not yet documenting MedRec on transfers. ${ }^{13}$ While studies have documented medication discrepancies based on MedRec, noted suboptimal communication strategies around medication management, or offered commonly held views as to why MedRec is not reaching its goals, ${ }^{4-9}$ no studies were identified in our literature review that carefully detailed current processes to understand barriers preventing success, or to document resource usage.

At least seven systematic reviews summarise randomised controlled trials (RCTs) and non-RCTs examining the impact of MedRec, either alone or as part of a more comprehensive medication management intervention on reducing adverse drug events and healthcare usage across multiple settings (hospitals, primary care, longterm care).$^{10-16}$ The conclusion of each review is remarkably consistent - well done MedRec reduces unintended medication discrepancies but the impact on clinical outcomes is uncertain. After restricting studies to those with a lower risk of bias, there is no evidence of beneficial effect on mortality or hospital readmission, although lowquality evidence suggests a trend towards decreased emergency department (ED) contact. ${ }^{15}$ This has led to a strong recommendation that medication reviews be undertaken only as part of a well-designed randomised trial, and include expert physicians able to directly determine and implement appropriate changes. ${ }^{15}$ Given that MedRec is carried out primarily to inform and improve the quality of medication review/management, these cautions bear heeding given the many competing priorities for healthcare resources. Meanwhile, resources (largely pharmacy and medicine) are being deployed to meet the forthcoming accreditation demands for widespread MedRec at all hospital transitions. Even if MedRec has a small and as yet unproven benefit on clinical events or resource usage, its cost-effectiveness may well be highly unattractive. Understanding the actual value for money spent for MedRec compared with competing priorities requires a detailed knowledge of the processes and people involved in those processes.

Usage of process mapping techniques in the context of healthcare is a relatively recent concept. The Multi-Center Medication Reconciliation Quality Improvement Study (MARQUIS) strongly recommends process mapping (a toolkit component) to understand the current state of MedRec processes and to perform gap analysis. ${ }^{17} 18$ Until now, only one study has used a process mapping technique to address MedRec. An evaluation of four community hospital EDs in Washington identified common problems that inhibited high-quality and efficient MedRec, which led to the development of an improved workflow. ${ }^{19}$ However, the process mapping was only done in focus groups, so represented what clinicians believed to happen, rather than what was actually observed to happen.

We set out to directly observe, document and evaluate local MedRec processes for internal medicine patients from ED triage to discharge. Our objective was to identify gaps in efficiency and safety, and to provide recommendations to improve the process.

\section{METHODS}

The study was based at the three hospitals containing internal medicine clinical teaching units (CTUs) in Hamilton, Ontario, Canada. Each hospital is affiliated with McMaster University's Faculty of Health Sciences, and each maintains quality of care and patient safety committees. The CTUs are managed by the service of general internal medicine (GIM) and staffed by Royal College-certified specialists and their trainees. At the time of the study (early 2014), GIM received 400-500 new patient referrals each month from ED physicians at each of three sites. The mean age of these patients was $\sim 70$ years; more than $90 \%$ were admitted with $74.2 \%$ of admissions occurring in 'non-business' hours between 19:00 and 07:00 on weekdays or anytime on weekends. Clinical pharmacy resources at each of the three hospitals include non-prescribing pharmacists supported by pharmacy technicians, fewer than one per medical team, and with coverage only during daytime hours.

In preparation for the process mapping, a large multidisciplinary study team including physicians, pharmacists, nurses, administrators and researchers representing each site met regularly to discuss local challenges and opportunities for improvement of MedRec. This revealed that each hospital had different MedRec processes, largely dependent on the availability of pharmacy resources to the EDs and the inpatient internal medicine CTUs. This varied from a pharmacy technician-led collection of BPMH after admission for seniors, to pharmacist-led MedRec only when requested by a physician or nursing staff. Since perceptions of activities, roles and coverage might vary from reality, we engaged an external process mapping private consultant group. This group provided three observers to simultaneously observe MedRec processes at each site consecutively for a week each.

The process mapping stage of the project was completed following stepwise strategy as summarised below.

\section{Step 1: Defining patient values}

Patient value statements were defined based on literature reviews, conversations with front-line staff and 
patients, and the consultants' experience in the industry. A value statement described what a patient values in the process, to ensure that improvement interventions are aimed towards increasing patient satisfaction in the process.

\section{Step 2: Mapping the processes}

The full current process was mapped by shadowing front-line staff in order to document all MedRec-related activities from ED triage to discharge. The goal was to capture what actually happened in the process as opposed to what was supposed to happen, or what clinicians think happens. The processes were observed long enough to see that the same steps occur multiple times. Actions which happened $80 \%$ of the times were considered the norm, and the less frequent actions were recorded as 'variations' in the process. While observing, questions were regularly asked of the staff to clarify the details of the work being done. Tasks were timed by observation when possible. When available, relevant process statistics, such as hours of staff availability, activity volume and frequency, and number of staff available were collected. As a last resort, if a step or action could not be observed, information was collected through a staff interview during the observation period. At the end of each day, the three process mapping consultants met to draft maps and resolve discrepancies, continuing over several more days.

\section{Step 3: Validating the process maps}

The completed process maps were presented as large physical posters at workshops and day-long open house sessions at each of the three hospitals, with invitations targeting the hospital staff who performed or were directly involved with management of the MedRec-related tasks. Feedback and change ideas were encouraged from all, particularly those directly engaged in the MedRec-related processes on a daily basis. All feedback was tabulated, and suggested corrections to current processes were validated by members of the research team, then incorporated into the final maps.

\section{Step 4: Planning change}

Draft process maps and key observations were reviewed with the research team using a grounded theory approach, to develop key themes of gaps in the processes. The final maps, key observations and change ideas were then presented to the study team as well as healthcare professional and patient safety leads from all three sites. Value-added-activities were identified as those currently undertaken which contributed to the patient value statements (see the Step 1: Defining patient values section).

\section{RESULTS}

Directly observed care took place sequentially across the three sites in the last quarter of 2013, with the final
Drafting Change process taking place in early 2014. Fifty-two staff members provided feedback on the draft process maps. Seven final process maps, each describing 5-14 different processes, were created for each of the three hospitals. The relationship of these process maps is shown in figure 1 .

\section{Medication information gathering: on admission}

Medication history was collected several times from triage in the ED to the time that patients were admitted to the ward (CTU) - several times by ED clinicians, then by internal medicine house staff and occasionally by attending physicians. This mostly took $1-5 \mathrm{~min}$ each time; variable methods and sources were used (eg, patient, other source such as the Electronic Health Record (EHR) or Ontario Drug Benefit Drug Profile Viewer (ODB profile)), partial information was frequently collected (eg, medication names only), and there was considerable duplication of information collected. A final admission MedRec, performed by pharmacists with or without assistance from pharmacy technicians, was considered the 'gold-standard' BPMH.

The capacity to complete the gold-standard BPMH was limited (maximum 60\% of all admitted GIM patients) and thus missed many patients. The pharmacy BPMH took 45-60 min of activity, but delays waiting for return communications from community pharmacy providers were frequent, at times limiting the timeliness of the completion. Once completed, it was the most detailed, including multiple sources, but then was erratically communicated to the patient's medical team. Usually, the BPMH could be found on paper somewhere in the patient's chart, occasionally in the EHR, or discrepancies were noted in the Orders section. Each pharmacist involved in producing BPMHs noted a lack of confidence that their output was routinely reviewed by the patient's medical team. Physicians noted the lack of standardisation around which patient received a BPMH, difficulties in locating one if it was carried out, and confusion regarding the best approach to a list of medication discrepancies.

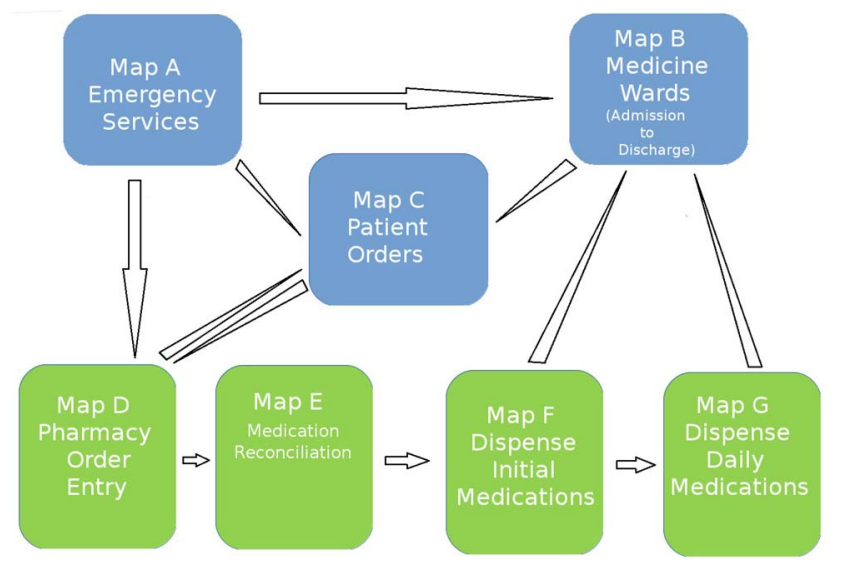

Figure 1 Medication reconciliation process maps. 


\section{Medication information gathering: on discharge}

Collection of medication information for the purpose of issuing the discharge prescriptions was carried out entirely by physicians and took $\sim 15 \mathrm{~min}$ on average. One hospital generated a templated discharge prescription electronically for review, a process favourably regarded by medical and nursing staff as time-saving and improving legibility. Sources used for the discharge prescription were mainly the Medication Administration Record (MAR), seldom included a BPMH and rarely received any input from a pharmacist. Notably, the MAR represents only the current medications being administered, and does not include those preadmission medications discontinued or held during the admission or those medications administered early in a hospital stay but stopped predischarge.

\section{Sources of medication information: reliability and completeness}

Multiple sources of medication information were identified as being used to assist with the MedRec process. The long-term care MAR was considered the most reliable and complete source of medication information, but only a small proportion of patients were admitted from longterm care. Many team members believed that the patient's blister pack or plastic pill box represented a 'gold standard', not realising that many medications that patients take ('as needed' medications or over-the-counter) are not included in these packs. Similarly, the community pharmacy history, patient's medication bag or written list, ODB profile, emergency medical services/ambulance crew record, the most recent discharge summary from a prior hospitalisation, patient's family and family doctor medication profile were all used as sources of medication information, but acknowledged to be potentially inaccurate or incomplete.

\section{Key findings}

Issues identified with the MedRec process were categorised into five themes.

1. Lack of use of all available medication history sources Physicians and nurses seldom used valid sources when collecting initial admission medication histories, primarily due to the source (community pharmacy profile in particular) not being readily available. No automated (computerised or fax-based) process exists to obtain the community pharmacy profiles during daytime hours or through the evening, night and weekend hours when a very large proportion of patients are admitted (nearly $75 \%)$ and community pharmacies are closed. At the time of discharge, physicians routinely failed to refer to the BPMH if there was one, or to the admission medication history to reconcile all medications. As a result, the discharge medication prescription tended to only reproduce the hospital MAR, missing several medications, failing to clarify changed doses or providing unnecessary duplication where a hospital auto-substitution had been made.

\section{Duplication of efforts}

The medication information collected in the ED (by ED physicians and triage nurses) was not trusted later in the process since it commonly lacked sufficient breadth and depth. It was frequently not referenced by providers downstream. Therefore, patients were asked for their medication history information 3-8 times at different stages of the MedRec process by different clinicians (triage nurse, triage physicians, receiving ED physicians, internal medicine physicians, internal medicine nurse and the pharmacist). Overall, this led to considerable inefficiency in the use of expensive healthcare professional resources-an estimated 1-2 hours per patient. Patients were frustrated by repeating the same information, but no patient carried a completely accurate medication list with them.

3. Inconsistent and untimely involvement of pharmacy The inconsistent and delayed involvement of pharmacy staff in performing BPMH was noted at each site. One site's pharmacy leadership, which had initially believed that all targeted seniors had a BPMH carried out at admission, realised through the process mapping that many patients admitted on weekends or evenings never received an admission MedRec. At all sites, limited pharmacy staff availability coupled with the time-consuming process of MedRec was noted to be a main cause. Lack of pharmacy services outside of weekday business hours and the subsequent weekday morning backlog were observed to exacerbate this issue.

4. Lack of standardisation and automation of the process

Lack of standardisation and automation of the MedRec process was noted. For example, physicians used highly variable queries to ask about medications, accurate synchrony between the MAR and the EHR at two sites was rare, and pharmacy staff BPMHs were frequently delayed by lack of staff, community information pending or awaiting a pharmacist's review and approval. This resulted in a maximum of 4-10 admission MedRecs per weekday depending on the site, or $\sim 21.8-49.3 \%$ of the average weekly admission rate for GIM.

5. Inadequate and inconsistent communication between staff

The lack of a formal process for consistent communication between the pharmacy and the rest of the care team was observed. This, coupled with the rare use of existing modules to track BPMH and medication profiles in the hospital's electronic system, was observed to result in miscommunication about completion of BPMHs and MedRecs. Furthermore, the current electronic systems do not consistently track the sources of medication information collected in a BPMH, which would provide information on reliability and completeness. Paper BPMHs were placed in the chart in varying places, and were not part of orders waiting for co-signature by a physician. Finally, the overly general use of the term 'medication reconciliation' to mean $\mathrm{BPMH} / \mathrm{admission}$ reconciliation, in-hospital MedRec or discharge 
reconciliation was further seen to result in miscommunication among staff.

\section{DISCUSSION}

We believe this to be the first description of the use of professional process mapping to identify problems, inefficiencies and suggest priorities for change in MedRec practices from admission to discharge in large academic teaching hospital settings. The rapidly deployed and completed observation period avoided the influence of 'secular change' confounding the results. We recommend several key changes to mitigate each of the problem themes that we identified.

First, improving the timeliness of the pharmacy-led $\mathrm{BPMH}$, to be completed as soon as possible when a potential admission is identified. This would require additional pharmacy staff or restructuring of current activities or further prioritisation of patients who require a BPMH. To assist, an automated system of secure request and receipt of community pharmacy profiles, as well as expansion of the coverage of patients included in the province's Drug Profile Viewer, is recommended. ${ }^{13}$

Second, a standardised record, and location for the $\mathrm{BPMH}$, as well as a mandated co-signature by the patient's medical team, is recommended. An accurate BPMH available at the time of the patient's admission could become part of the admission orders, improving timeliness and accuracy.

Third, computerisation of the MAR (present at one site), although not measured in this study, was judged to be a major time-saver for physicians completing a discharge prescription, given that the CTU patients are frequently taking more than 12 medications. However, a mandatory display and assessment of preadmission medications would also be required for accurate discharge MedRec.

Fourth, it was noted that patients or their caregivers are rarely aware of the changes made to their medications through the hospital admission to discharge process. While patient and caregiver education was felt to be valuable throughout, given the lack of resources available, it was decided that it should be focused on discharge where self-management was going to be key. This is now mandated at two hospitals as part of a discharge checklist.

Our study has several limitations. It is possible that not all actions relevant to MedRec were observed during the observation period, and hence the process maps may not be all-inclusive. In addition, we only studied one large Canadian academic health sciences system with three teaching hospitals. While internal medicine CTU activities are regularly audited nationally for quality and consistency, we cannot be sure that other hospital settings face similar challenges. Besides, this study does not address the crucial period of postdischarge medication management, a period where medication safety issues are known to spike. ${ }^{20}$ This is a topic for an ongoing study. Finally, the process mapping exercise is a necessary but not sufficient first step in understanding the resource usage applied to MedRec which comes with an opportunity cost. The relative value of MedRec, compared with competing patient care activities, remains a priority question.

On the basis of this study, minor changes in processes have occurred locally to improve the efficiency of MedRec. Current research is focused on the postdischarge medication management by patients with their community pharmacist and family doctor, as well as detailed costing of the MedRec process to better understand resource usage, and methods of prioritising which patients will most benefit from MedRec.

\section{CONCLUSION}

Our process mapping study contributes valuable information towards improving the quality and efficiency of the hospital MedRec process and towards more accurate measurement of its health resource usage and costs. High-quality studies to answer the question of costeffectiveness are still required.

\section{Author affiliations}

${ }^{1}$ Division of Clinical Pharmacology \& Toxicology, McMaster University, Hamilton, Ontario, Canada

${ }^{2}$ Department of Medicine, McMaster University, Hamilton, Ontario, Canada

${ }^{3}$ St Joseph's Healthcare \& Hamilton Health Sciences, Hamilton, Ontario, Canada

${ }^{4}$ Department of Clinical Epidemiology \& Biostatistics, McMaster University, Hamilton, Ontario, Canada

${ }^{5}$ St Joseph's Healthcare Hamilton, Hamilton, Ontario, Canada

${ }^{6}$ Hamilton Health Sciences, Hamilton, Ontario, Canada

${ }^{7}$ Clinical Pharmacology \& Toxicology, St Joseph's Healthcare Hamilton, Hamilton, Ontario, Canada

${ }^{8}$ Doleweerd Consulting Inc., Orillia, Ontario, Canada

Contributors $\mathrm{AH}$ led the conception of this study and acquisition of funding, participated in acquisition and analysis of data, and developed the final manuscript. JMB assisted with the design of the study, analysis and interpretation of the data and revision of the manuscript. HP, CO, JJY, DP, CN and AP assisted with the design of the study, acquisition and interpretation of data, and revision of the manuscript. RT assisted with the analysis and interpretation of the data and drafting and revision of the manuscript. JD and TB led the design of the study, acquisition of data, and assisted with developing of the manuscript. ST assisted with the design of the study, acquisition of the data and revision of the manuscript.

Funding Ontario Ministry of Health and Long Term Care Academic Health Sciences AFP Innovation Grant (Award number HAH-13-007).

Competing interests JD and TB are employees of a private consulting company hired to carry out the process mapping.

Ethics approval The study was approved by the Hamilton Integrated Research Ethics Board (Application numbers 13-841 and 13-842).

Provenance and peer review Not commissioned; externally peer reviewed.

Data sharing statement Additional data are available by emailing $\mathrm{AH}$ at holbrook@mcmaster.ca.

Open Access This is an Open Access article distributed in accordance with the Creative Commons Attribution Non Commercial (CC BY-NC 4.0) license, which permits others to distribute, remix, adapt, build upon this work noncommercially, and license their derivative works on different terms, provided 
the original work is properly cited and the use is non-commercial. See: http:// creativecommons.org/licenses/by-nc/4.0/

\section{REFERENCES}

1. Accreditation Canada. Required Organizational Practices Handbook. 2014. https://accreditation.ca/sites/default/files/rop-handbook2014-en.pdf

2. Urban R, Armitage G, Morgan J, et al. Custom and practice: a multi-center study of medicines reconciliation following admission in four acute hospitals in the UK. Res Soc Adm Pharm 2014;10:355-68.

3. Accreditation Canada, the Canadian Institute for Health Information, the Canadian Patient Safety Institute, and the Institute for Safe Medication Practices Canada. Medication reconciliation in Canada: raising the bar-progress to date and the course ahead. Ottawa, ON: Accreditation Canada, 2012. https://accreditation.ca/sites/ default/files/med-rec-en.pdf

4. Wanbon R, Lyder C, Villeneuve E, et al. Medication reconciliation practices in Canadian emergency departments: a national survey. Can J Hosp Pharm 2015;68:202-9.

5. Vira T, Colquhoun M, Etchells E. Reconcilable differences: correcting medication errors at hospital admission and discharge. Qual Saf Health Care 2006;15:122-6.

6. Pevnick JM, Shane R, Schnipper JL. The problem with medication reconciliation. BMJ Qual Saf 2016;25:726-30.

7. Manias E, Gerdtz M, Williams A, et al. Communicating about the management of medications as patients move across transition points of care: an observation and interview study. J Eval Clin Pract 2016;22:635-43.

8. Sponsler KC, Neal EB, Kripalani S. Improving medication safety during hospital-based transitions of care. Cleve Clin J Med 2015;82:351-60.

9. van Sluisveld N, Zegers M, Natsch S, et al. Medication reconciliation at hospital admission and discharge: insufficient knowledge, unclear task reallocation and lack of collaboration as major barriers to medication safety. BMC Health Serv Res 2012;12:170.

10. Lehnbom EC, Stewart MJ, Manias E, et al. Impact of medication reconciliation and review on clinical outcomes. Ann Pharmacother 2014;48:1298-312.

11. Kwan JL, Lo L, Sampson M, et al. Medication reconciliation during transitions of care as a patient safety strategy: a systematic review. Ann Intern Med 2013;158(5 PART 2):397-403.

12. Mueller SK, Sponsler KC, Kripalani S, et al. Hospital-based medication reconciliation practices: a systematic review. Arch Intern Med 2012;172:1057-69.

13. Chhabra PT, Rattinger GB, Dutcher SK, et al. Medication reconciliation during the transition to and from long-term care settings: a systematic review. Res Soc Adm Pharm 2012;8:60-75.

14. Bayoumi I, Howard M, Holbrook AM, et al. Interventions to improve medication reconciliation in primary care. Ann Pharmacotherapy 2009;43:1667-75.

15. Christensen M, Lundh A. Medication review in hospitalised patients to reduce morbidity and mortality. Cochrane Database Syst Rev 2016;2:CD008986.

16. CADTH Rapid Response Review. Medication reconciliation at discharge: a review of the clinical evidence and guidelines. 2012 https:/www.cadth.ca/media/pdf/htis/april-2012/RC0339\%20-\% 20Medication\%20Reconciliation\%20Final.pdf

17. Salanitro AH, Kripalani S, Resnic J, et al. Rationale and design of the Multicenter Medication Reconciliation Quality Improvement Study (MARQUIS). BMC Health Serv Res 2013;13:230.

18. Mueller SK, Kripalani S, Stein J, et al. A toolkit to disseminate best practices in inpatient medication reconciliation: Multi-Center Medication Reconciliation Quality Improvement Study (MARQUIS). Jt Comm J Qual Patient Saf 2013;39:371-82.

19. Hummel J, Evans PC, Lee H. Medication reconciliation in the emergency department: opportunities for workflow redesign. Qual Saf Health Care 2010;19:531-5.

20. Forster AJ, Clark HD, Menard A, et al. Adverse events among medical patients after discharge from hospital. CMAJ 2004; 170:345-9. 